\title{
The opacity of play: a reply to commentators
}

\author{
C. Thi Nguyen \\ (This is my reply to commentators in the Journal of the Philosophy of Sport's \\ special issue symposium on my book, Games: Agency as Art. \\ This is a pre-print. Please cite the final version at: \\ https://www.tandfonline.com/doi/full/10.1080/00948705.2021.1993870)
}

First, let me thank all my wonderful commentators, and the editors at the Journal of the Philosophy of Sport for this extraordinary opportunity. I had harbored suspicions, as I worked away on Games: Agency as Art, that I was pouring my heart out into a hopelessly niche product, borne of my own private obsessions. I am so grateful to have this bounty of interest and commentary from so many walks of philosophy.

It is quite tempting, in a situation like this, to try and defend myself over every jab and criticism, but the result would be miserably compressed and spiritually impoverished. Instead, I will cover a handful of key points in detail. Much of the commentary clusters around a few topics, indicating some intellectual centers of gravity for my readers' concerns. I will focus on these clusters; I apologize to my commentators for all the other exchanges that will fall by the wayside.

The three centers of gravity I take to be:

1. Is striving play really as absorbed and pure as I make it out to be?

2. Is striving play really as valuable as I make it out to be?

3. Is there really an aesthetics of agency, and is it really so important for the gaming experience?

I will assume anybody reading this response is already at least ankle-deep in this material, and is familiar with the basic outlines of my account. In what follows, all bare page number citations will be to Games: Agency as Art; citations to others' articles in this symposium will include the author's name.

\section{The purity of striving play}

Several of my commentators raised a set of issues and examples centered around the same concern: is striving play really as pure and absorbed as I made it out to be?

A brief reminder: in my account, there are two forms of game-play, achievement play 
and striving play, which are distinguished by the motivational state of the player. Achievement play arises from an enduring interest in winning (or some other standard of achievement). Striving play involves adopting a temporary interest in winning, for the sake of the player's engagement in the activity of trying to win. In other words, striving player players adopt the goal of winning as a mere means; their real end lies in their engagement in the process of trying to win.

In some cases, in order to get the kind of engagement they want, the striving player needs to temporarily put their real purpose out of mind. That larger purpose might be, say, to have a certain kind of absorbed practical experience. To achieve that larger purpose, the player must absorb themselves in a temporary inner agency that is wholly devoted to the win. They need to form a layered agency, one which walls off their larger purpose, excluding it from their in-game practical reasoning. Let's call this "opaquely layered striving play" - or "opaque layering" for short.

Opaque layering is supposed to be an extreme case of striving play, though an extremely interesting one for theorists of agency. To be perfectly clear: I never claimed that opaque layering is common, easily achievable, or necessarily involved in all valuable game-play. The point of the arguments in the book is to show that opaque layering, in its most fully opaque form, is possible, and at least occasionally actual - most particularly, in players who want to be totally absorbed in an instrumental struggle. Some of the clearest examples of opaque layering are in people who engage in intense competitive play - like tournament players of Chess, soccer, Magic: The Gathering, and Starcraft 2 for a love of the absorbed struggle. I focused on this extreme case for a few reasons. First, I was trying to demonstrate the possibility of that kind of agential layering and agential fluidity. Second, it seemed easier to start with the purest case, at the far end of the spectrum, and then later to backtrack and think about more muddled cases. In fact, I suspect that many everyday cases of striving play involve partial, rather than total, opacity - so they will inherit some, but not all, of the characteristics of fully opaquely layered play.

But a number of my commentators have criticized my picture of opaquely layered agency. They question how common it is, or how accurate a description it is of actual everyday game-playing. Here's one way to put the issue. As Stephanie Patridge notes, the opaque striving player and the achievement player should behave identically inside the game. The achievement player actually wants to win. But the opaque striving player temporarily absorbs themselves inside an inner agent who is entirely devoted to winning. So the salient controlling agency during the game should be motivationally identical between the opaque striving player and the achievement player. In fact, this kind of striving player is essentially trying to simulate being an achievement player. They want the kicks of deeply caring about the win without actually becoming the kind of person who cares enduringly about winning. But, in order to get what they want, they will have to become something almost indistinguishable from an achievement player - at least, 
during game-play.

This is my theoretical image, at least, of a certain type of striving player. But Patridge doubts that this kind of striving play actually occurs all that often in normal circumstances. A number of my other commentators have also pushed back against this theoretical image by presenting examples where the striving player and the achievement player end up behaving quite differently during play. William Morgan, for example, admits that he lacks a certain "killer instinct" for certain games, like racquetball. This he takes to show that he is a striving player. And it changes the way he plays racquetball: "giving my opponent the benefit of the doubt on whether, say, a serve was in or out, and/or refraining from overly exploiting weaknesses I detected in my opponent's game, and, more generally, trying to create a festive, fun game environment to discourage hypercompetitive behavior". In this case, we can say that Morgan's larger purpose permeates his in-game agency - which, provided we assume that Morgan is a striving player, runs against my description of opaque layering.

Adrian Currie provides a particularly crisp example of such permeability. Imagine, says Currie, that we are all at a party and decide to play a round of that profoundly stupid game, Bag On Your Head, to "much hilarity and hijinks." Because it is a stupid game, the players must surely be engaged in striving play. (Recall: according to my account, in such a stupid game, our purpose is to experience the hilarity of failure. But in order to do so, we have to actually pursue the win with some degree of fervor. We can't fail hilariously unless we were actually trying to succeed.) The whole thing, observes Currie, should be played with a lighthearted air. But suppose we have a rich friend who detests the silliness of Bag On Your Head. Perhaps, as Currie suggests, our rich friend is an "obstinate achievement player," unable to give themselves over to the fun of silly striving play. So our rich friend decides to change the nature of the activity by offering a prize. For the second round, our rich friend promises to give away their incredibly expensive collection of Faberge eggs to the winner. Obviously, the addition of this prize will change the mood of the game. The players will, in all likelihood, become far more serious about winning. And this induced seriousness, says Currie, will undermine the experience of joy, lightness, and fun in the game. The new stakes will prevent the players from being playful.

I find this example particularly enlightening. Currie never presents this as an open criticism of my view, but it could easily be taken that way. It presses with surgical precision at the exact relationship between larger purpose and inner agent. In my model, the fully opaque striving player and the achievement player should behave identically during the game. But Currie is describing a case precisely were where the introduction of an external reward forcibly shifts the players from striving play to achievement play and then that shift changes the manner and spirit of the striving play. That latter shift couldn't have happened unless the larger purpose can penetrate into the inner agent and change how they play the game. Currie's example only makes sense if achievement players play with a more deathly grip on the win, and striving players play more lightheartedly. Any account 
of striving play must surely explain the phenomenon of permeability. But does my account have the space for it?

First, let me insist on one simple thing: the existence of permeability, in this case, doesn't weigh against the bare point I was pursuing in the book. There, I only wanted to show that such opaque layering was sometimes possible. (This is likely why Currie doesn't present this example as an outright criticism.) The simplest defense of my position would be to admit that these examples of permeability are properly described, but point out that they don't exhaust the landscape of striving play. I could say: sometimes, we certainly do engage in striving play in a more transparent and permeable manner. But other times, we do screen off our larger purpose from the inner agent. And the best examples of the latter are not found in social examples of casual play, but in the kind of intense, absorbed, totally committed play we find in, say, serious tournament play - in the Olympics, in Magic: The Gathering tournaments, and in Starcraft 2 tournament e-sport play. There, the striving player and the achievement player do seem motivationally identical during play. They play just as intensely, and just as seriously, with just as much total devotion to the win. For these opaque striving players, adding stakes won't change anything, because they were already playing with maximum seriousness - but for striving reasons.

For such players, we may only able to detect the difference between striving players and achievement players in their extra-game behavior. Patridge supplies an excellent example: when a professional athlete quit their sport, even when they were winning lots, because it just wasn't enjoyable for them. Such an athlete has revealed themselves to be a striving player. After all, a pure achievement player who is continuing to rack up the wins - but not enjoying themselves at all - has no reason to quit the game. They're in it for the achievement, not some experiential quality of engagement. Quitting something you're good at simply because it isn't fun only makes sense for players with a striving motivation.

So: I grant all these examples of non-opaque striving play, and only point out that I had never denied its existence. I was simply most concerned with showing the possibility of opaque striving play, too. But I want to emphasize here, the importance of these examples and of my commentators' insistence on this point. They point to a clear lacunae in my account, thus far. In the book, I had carefully modeled the most extreme sort of opaquely layered striving play. I had not yet worked out in detail the kind of permeable striving play present in ordinary social cases, and that surely deserves filling out, too. My mea culpa: the concepts of striving play and agential layering are sufficiently unusual, at least in philosophy, that I had to spent most of my time just demonstrating that it was possible and real. And for that purpose, the purest form was easiest to work with. But there's certainly room for both opaque layering and translucent layering in the wide world of play.

Let's now turn to a slightly different worry: a worry about in-game adaptability. Patridge argues that a certain kind of achievement player will be socially and morally 
better than the striving player, as I have describe them. Imagine, she says, an achievement player with mixed motives, who plays partly to win and partly to have fun. They can "adjust their gameplay in situ, as it were," changing how they pursue the win in order to make it consistent with their other competing ends. But the striving player is unable to do so. Why? Patridge's argument is that we often have pro-social reasons to adjust how we play during the game. The achievement player with mixed motives can take those prosocial reasons into account as they play. But the pure striving player will be absorbed entirely in their alternate agency - which is wholly devoted to the win - and they will have screened off any larger motivation. So they have no motivation during play to alter their style of play to better support pro-social ends. Even if they originally set out to play for a social purpose, the opaque nature of striving play prevents them from being motivated by that social purpose during play.

If Patridge's argument works, it would be a striking condemnation of striving play. Because, obviously, mid-game adaptability is often crucial for pro-social play. Not only are we typically capable of such mid-game adaptability, it is clearly a superior way to play in many circumstances. Most of my boardgames nights with friends are played for social reasons, and I want everybody to have a good time. Here's a common situation: sometimes, when playing a familiar boardgame, a new player will commit an error in their first few moves that leaves them painfully vulnerable. If I take advantage of that error, I will gain some small advantage, but they will be utterly hamstrung from the get-go. They'll have to play the rest of the game knowing that they have absolutely no chance of winning, and from a position with so few resources that their gaming experience will likely be pretty miserable. But if I ignore their one opening error, then they can have an enjoyable and interesting experience. So: if my sole interest were in winning, I should take advantage of that early error. But almost always, in a social board gaming situation, the right thing to do is ignore that opening error. I do want to give novice players a chance to stretch their legs and figure out how the game works without hamstringing them at the first move. 1 So if opaquely layered striving players really were incapable of such adaptation, that would show that striving play lacked a capacity for a certain kind of social grace. And it would also show that opaquely layered striving play was even less common than we thought, given the frequency with which we do see adaptability in actual gameplaying.

First, let me note that, even if all of Patridge's criticisms succeed, that doesn't weigh against the claim that perfectly opaque layering is possible, and sometimes real. But I don't think that's all I can say. I think, in fact, that opaque layering is compatible with adaptability, if we impute at least one more ability to the layered agent.

The hidden presumptions, in Patridge's criticism are: first, that a opaquely layered

1 Notice that, on the other hand, punishing an early error like this is usually the right and normal course of action in, say, a chess tournament or on most online chess servers. One minor reason for this may be that in two-player abstract boardgames, a player who has lost early may simply resign, and move on to the next game. Resignation is normally not a workable solution in multiplayer boardgames 
striving player will stay in the inner layer for the entire game; and second, that they must use only a single unchanging inner agent per game. If we accepted those presumptions, then we should expect the striving player to be unable to adapt during the game. But there is no particular reason to think our agential capacities are limited in such a way. Imagine, instead, an opaquely layered, but flickering agent - one who usually operates in a mode of opaque layering, but with the capacity to pause mid-game, pop from the inner agent's perspective to the outer agent's perspective, and modify their inner agent, before popping back in. Such a player could both maintain opaque layering during play, but also sometimes adapt to other considerations, by occasionally interrupting their opaque absorption.

The flickering (opaquely layered) striving player is such that:

1. When they are engaged in striving play, they are opaquely absorbed in their inner agent.

2. At intervals during play, they briefly switch perspectives from the inner to the outer agent.

3. During these intervals, they inspect how play is going from the perspective of their larger purpose.

4. They have the capacity, during these intervals, to modify their inner agent, before plunging back into it.

The flickering agent can, in that case, modify how they are playing in response to the larger purposes at hand, while they are occupying the larger perspective. The flickering agent can step back and notice that a novice player is struggling painfully. The flickering agent can alter their inner agent. The flickering agent might decide to give themselves a handicap, or ignore a certain kind of opportunity, or put a certain kind of stratagem outof-bounds. The flickering agent can then enter this as a new limitation on how they play the game; they can modify the inner agent before they re-absorb themselves back inside it. In other words, they can pop their head up for a moment, and decide to play a slightly different game.

I should make clear here that, though I think that this description of the flickering agent is compatible with my account in the book, this description is very much a new addition - and I owe a debt to Patridge for pushing me to articulate it.

Is my description of the flickering agent merely some legalistic dodge? I don't think so. The wholly transparent agent plays constantly in light of, and is constantly responsive to, their larger purposes. The flickering agent, on the other hand, spends most of their playtime disconnected from their larger purposes. Those moments of re-connection to their larger purposes are confined to certain moments, where they mentally step back from the game and assess. The flickering agent is an opaquely layered agent, but with the added capacity to pop out to their larger perspective, mid-game, and modify their inner 
layer.

I had originally postulated agential layering as a response to problems arising from the pursuit of self-effacing ends. If relaxation is a self-effacing end, then we can't achieve it by pursuing it; we need to put that end out of mind to achieve it. Keeping that end in mind would undermine our pursuit of it. Notice: a non-flickering opaque striving player can agent can pursue self-effacing ends, but they cannot adapt mid-game. Patridge's mixedmotive achievement player, on the other hand, can adapt mid-game, but cannot pursue self-effacing ends. The flickering opaquely layered agent can both adapt mid-game and pursue self-effacing ends. The flickering agent can use opaque layering striving to get absorbed game-play experiences, while at the same time maintain the capacity for responsiveness to extra-game considerations, during their flickers. They just need occasional access to a pause button on their opaque layering. And this description of the flickering agent rings true, at least of my own experience of game playing.

Let me be clear: my claim is not that all mid-game adaptation is best described in terms of a flickering opaque agency rather than a wholly transparent one. My claim is only that they are both good explanations of adaptation, and, I think, both really occur. From my own game-playing phenomenology, I would say that my agency is usually steadily transparent when I am teaching a game to total novices, or where I am much more concerned with the social impact of the game. And that happens often, like when I am more concerned with my role as party host than in my own pleasures of absorption in the game. But I would say that my agency is typically more of a flickering agency in more gaming-focused contexts. I think my usual boardgame night with my gaming group, who are all fairly intense players, is more characterized by a flickering agency. There, most of my experience is in absorption in the struggle to win, with the occasional pop-out to check in to see if the others are doing OK. And sometimes that observation will create a modification of play: for example, if I notice that one player's in-game economy is wholly busted, and that they are flailing and particularly not enjoying themselves, I may decide to refrain from further abusing their vulnerable position to garner extra resources, and then go back to playing the game hard - with that one added constraint.

My point is not to deny the possibility of more transparent forms of gaming agency. My point is that the observation of mid-game adaptability does not, by itself, force us to postulate a fully transparent agent. Such adaptability can be explained in at least two ways: by a fully transparent agency, or by an opaquely layered agency that intermittently interrupts itself to scan the terrain from the larger perspective.

One might now ask: what generates that interruption? That is, if I, as a striving player, am capable of interrupting my absorption in winning in light of some threat on the larger purpose - then must I not be in some degree of touch with the larger purpose throughout?

First: not necessarily. A game-player might develop the habit of periodically popping their head up, stepping back for a moment back into their outer agency, and checking up 
their progress toward the larger purposes. But this is very different from a player who is constantly operating with some awareness of the outer agency's purposes. I think this is quite familiar. In boardgames, while somebody is still taking their turn, I'll sometimes take a bit of a mental breather. I've done all the calculations I can, so I lean back and take a deep breath. This is when I notice that I'm thirsty or need to pee, or that somebody else at the table needs a drink, or that another player seems utterly miserable. (Such moments of stepping back do arise more naturally within the typically slower pace of boardgames, which might explain why I have engaged more often in regretfully assholish behavior during fast-paced online shooters than I have in slower-paced boardgames.)

But I think often what's going on is even more complicated than that. Often, we don't first initiate a breather and then notice that something has gone wrong. Sometimes, we notice immediately during game-play that something has gone wrong - that some player is unhappy, or miserable, or offended - and that noticing is what pops us out of our absorption. This is usually how it goes with cases like the novice's early-turn move. Even if it's my turn and I'm currently absorbed in trying to win, I am capable of noticing that my optimum move would completely destroy another player's entire gaming experience - and I am capable of flagging that as a problem, from the perspective of my larger purposes. If that happens, then the outer layer must have been monitoring the goings-on to some limited degree

And I think that's actually the best description of what's going on in many instances of game-playing. I don't think I've described it adequately here; our theoretical models for agency are presently impoverished, hereabouts. The best I can do is to reach for a rough analogy. In many games, I suspect, our enduring agency works something like the operating system on a personal computer. It's still running in the background, even as some specific program dominates most of our phenomenal vision. But the background system is still capable of noticing certain problems and stepping in and taking over. It's also capable of lying in the background until certain pre-arranged triggers wake it up like phenomenological hot-keys. If I'm right, then something very complicated is going on with our agency. We have the ability to set up layers and run them simultaneously in parallel - where one layer takes up the foreground of our conscious awareness, but the other is hanging out in the background, able to notice certain triggers and intercede.

But notice that such a complex structure - primary absorption in an inner layer, with a background layer running background checks - is a very different structure from an utterly transparent form of agency. If one is utterly transparent, then one's larger purpose would loom as large within the game as without. But the utterly transparent agent runs into all the problems I described in the book. Often, we have collective fun only by devoting ourselves to the goal of winning the game. So keeping the goal of collective fun constantly in mind undermines our ability to achieve it. The transparent agent is caught by such examples. This more complex agent I've described - the flickering agent with multiple agential layers, one dominating in the foreground, the other running quietly in 
the background - is capable of dodging such problems, because they have the capacity to exclude their larger consciousness from the forefront of their mind for long stretches of game-playing. And I think this description is phenomenologically accurate of many of our gaming experiences. Absorption in the attempt to win dominates the landscape of our consciousness, and our larger purposes recedes - but not so far that we aren't capable of noticing abject failures to achieve that larger purpose.

The kind of agency I'm describing might seems absurdly complex. But I think it is exactly the kind of agency that we need to handle all sorts of real-world problems in practical reasoning. There are many cases where it benefits us to focus on one kind of thing and mostly ignore the rest of the world, but where also we need to let other concerns break in when they reach some high threshold. 2 It is very useful for me to ignore all my other pressing concerns when I focus on writing philosophy or teaching. But the rest of interests need to be able to flood in if a sufficiently powerful trigger goes on - like the sudden sharp smell of smoke, or the fact that it's raining so hard that maybe I should worry about flooding in the house. 3 (I made a first gesture at this kind of possibility in the book, when I noted that even the most absorbed game players would interrupt their absorption if another player broke down crying, or if their house caught on fire (57). For a very detailed analysis of how this kind of focus might help us in non-game situations, please see Luca Ferrero's lovely extension of my account of agential layering in this issue.)

To sum up: I have granted the importance of the examples highlighted by my commentators, in which our larger purposes can change and inflect the mood in which we play. I happily grant that, in some cases, this occurs because the division between the layers is not perfectly opaque, and the purpose for which we play changes the feel of the inner agent. But in other cases, the best explanation of the phenomenon of adaptability is something more arcane: a flickering agent who occasionally raises their head out of their inner layer, or an agent with a complex two-part structure of foregrounded and backgrounded layers of agency, running simultaneously.

One more note: Emily Ryall observes that we can be stuck in a peculiar situation, when we are "stuck between layers: when our outer layer wants to pull us away but our inner layer wants to continue". Such is the case with grinding in games, when we find ourselves stuck doing something tedious because of the inner agent's desire to win, and unable to cancel the inner agent's grip on our motivations, even when the outer agent wishes to end. I have had this experience many times with various addictive video games - like my almost career-endingly problematic relationship with Civilization IV, which I am never allowed to touch again.

I agree entirely with Ryall's worry. Something has, indeed, gone wrong for us in such

2 Compare to Michael Bratman's (1999) account of intention, where an intention involves settling one's mind and defeasibly closing oneself to considering further options — unless, of course, something sufficiently big and disruptive happens .

3 The flood example actually happened while I was writing this article. My writing focus was so intense that I almost didn't notice the impending flooding of my house. Almost. 
cases. The inner agent was supposed to be a temporary construct, set up at the behest of our enduring agency, to serve our enduring ends. If the point of playing the game was to serve the purpose of having fun, then we should stop playing when it isn't fun anymore. To do otherwise is to display a form of weakness of the will. In this case, letting our temporary, instrumentally-derived ends grip us and drive us to act against our larger interests. It is a rational mistake for a striving to pursue their adopted in-game goals at the expense of their more enduring ends, when those in-game goals were adopted just for the sake of achieving those enduring ends.

But that way of putting it might seem a bit crude, once we have been through all the torturous observations about the way that we can foreground the inner agent. The whole point is that, to play in an absorbed way, sometimes we have to forget our enduring interests. So doesn't that open up a gateway for being stuck in the inner agent?

That worry, however, arises only when we abstract away much of the actual practice of game-playing. In normal gaming life, we occasionally step back from our inner agent. In most games, the game finishes - or at least pauses significantly - and we set aside the inner agent and return to the perspective of our more enduring agency. Even if certain gaming experiences demand opaque layering, we need only engage in such opacity during the game. The standard practice of game-play contains natural boundaries. Passing such a boundary creates a bit of natural breathing room, to ask if all that absorbed striving was worthwhile. We may occasionally go a little too far and engage in a bit of striving that is unjustified in terms of our larger purpose, in the moments between those built-in breathers. But the amount of that excess is delimited by the natural boundaries of typical game-playing. I might regret playing that last boardgame, or those last two hours of grinding through that video game, but once I hit one of those natural boundaries, I should realize that my larger purposes are unfulfilled. 4

I don't take those small-scale wastes of effort to be weakness of the will. They are the inevitable byproduct of the logic of absorbed striving. If the only way to get the pleasures of absorbed striving is to temporarily put yourself out of touch with your larger purposes - well, then, you'll pay the occasional costs that come from being temporarily out of touch with your larger purposes.

The cases which are really worrying are those in which one reaches a boundary, resumes one's larger perspective, and decides that this game is actually a waste of time - and then finds oneself compelled to keep playing that game anyways. Such was the case when I quit my current game of Civilization IV, shut down my computer, step away from my desk, and curse myself out for all those wasted hours, and swear to myself that I would delete the game - before immediately booting it up again and launching a fresh game. Those are the cases that seem like real weakness of the will, and which are significant candidates for counting as addiction.

4 This may go some way towards explaining the problem with pervasive gamification, as I discuss in Ch. 9. 


\section{The value of striving play}

Next, let's talk about the value of striving play.

Let me start with William Morgan, who worries that striving play isn't the glorious thing l've made it out to be and leaps to the defense of achievement play. In my original discussion, I tell a number of stories about my spouse and my life with games. Sometimes, I said, when we were lucky enough to find ourselves precisely matched, we can give ourselves entirely to competition within the game. But, says Morgan, my spouse and I could give ourselves up wholly to the game only because all the stars had aligned. My spouse and I are intimately aware of each others' strengths and weaknesses, have a shared desire for pleasurable competition, and are equally skilled at racquetball. For this reason, says Morgan, my spouse and I rarely need to take a reflective break.

But most of the time, says Morgan, the stars don't align so nicely. We have to play with people that don't share the same spirit or don't have the same skill level. So we are, says Morgan, "saddled by a double-mindedness". We have to reflectively step back and evaluate, to make sure things are going alright. But, says Morgan, this need for doublemindedness conflicts with another part of my view: the view that single-mindedness is part of the good-life. The striving player, under normal circumstances, cannot permit themselves that single-mindedness. A player interested in collective fun, for example, always has to be fussing and worrying about whether other people are having fun interrupting themselves, stepping back, and reflecting. To put it in the language we just developed above, even if the striving layer sets up a nice juicy layer to absorb themselves into, they will still have to frequently flicker out of it for pro-social reasons. Only the achievement player, says Morgan, can be utterly wholehearted. They have no reason to interrupt themselves and check if their attempts to win align with their true purpose, for winning is their true purpose.

However, I never claimed that single-mindedness was part of the good life. Rather, I claimed that an experience of single-mindedness was pleasurable and the basis for many aesthetic goods. But life often asks us to be double-minded - to be attentive to a thousand different values and considerations, and to balance them all. Life calls from us a complex, muddled, pluralistic, and qualified consciousness. Cultivating singlemindedness, as an enduring psychological state, in a world this morally complicated, is, I think, a profound vice. This is exactly why games are so special. Games offer us the ability for us to carve out a carefully delimited space for single-mindedness, thus gaving us access to some of the experiential pleasures and goods of single-mindedness - without our having to become actually, enduringly single-minded. They let us indulge our taste for single-mindedness, without giving ourselves over to it completely.

In fact, I take the finale of my book to be a defense of the value of double-mindedness, in the form of the playful attitude (216-24). Maria Lugones (1987) puts it this way: to be playful is to be able to shift between normative mindsets, to wear each one a little lightly. 
And I suggested that what is really important, for our moral development, is cultivating the ability to switch between temporary bouts of single-mindedness and a larger and messier perspective. We need to cultivate the capacity to focus intensely in one agential mode - on one goal, and one specific set of abilities to pursue it. But we also need to cultivate the capacity to interrupt that agential mode and step back from it, question it. Without that, I think, what we get is the world of cutthroat Wall Street financiers pursuing more profit endlessly, generals pursuing higher kill counts endlessly, and pick-up artists pursuing ever more one-night stands. 5 We get a world of unreflective devotion to standards of excellence. Where Morgan sees the nobility of single-mindedness, I worry about the unchecked spirit of raging competitiveness. Double-mindedness is a virtue precisely because it takes us out of the pleasures of pure absorption in a single goal, and provides a check on our tendency to lose ourselves in the unreflective pursuit of success on some pre-established terms. We should take our single-minded kicks when we can get them, when it's safe, but we should also take care to not let that attitude too far out of its kennel. And cultivating the interrupting, plural spirit of double-mindedness is, in my mind, something of an insurance policy. The value of striving play lies, in part, in its potential to cultivate double-mindedness, while simultaneously allowing us to indulge in the pleasures of single-mindedness.

Let me now turn to a very different worry. J.S. Russell worries that opaquely layered striving play is too close to achievement play and so inherits its chief ills. Suppose that competitiveness is, by itself, bad. Competitive games, says Russell, "require pursuit of greater comparative worth". This is problematic, suggests Russell, precisely because it is bad to measure one's worth against another person's, rather than against some noncomparative standard of perfection. And in striving play, we engage in such measurement, just as much as we engage in it with achievement play. So striving play does not give us any out, despite my arguments to the contrary in Chapter 8.

But I think this discussion fails to distinguish how different the evaluative process is between striving and achievement play. In achievement play, to the extent that we pursue comparative success, our ability to achieve that success - to be better than the other person - is part of our lasting sense of value in the activity. But in striving play, we pursue comparative success in an agentially bracketed way. We take on that care for a while, and then we slip out of it. Our evaluation of the value of the activity, from our larger and more enduring perspective, can focus on something other than comparative success: like aesthetic value, or fun, or collective social joy. A genuine striving player doesn't measure their worth against another from their enduring standpoint.

Let me try approaching the issue from another angle. Imagine two different worlds: a world full of achievement players and a world full of striving players. On first blush, it seems like the world of achievement players cannot get as much total value out of game-

5 This is a pointer towards a paper, in development, by myself and Olufemi Taiwo, on the relationship between an out-of-control gaming attitude and wartime massacres and pick-up artists. 
playing as can the world of striving players. Here's the simplest scenario. Suppose the value of achievements is inextricably linked to winning, or ranking, or some other comparative scale. It is impossible, in the world of achievement players, for everybody to come out the best. We can't all be winners - not, at least, with competitive games. But in the world of striving players, everybody can be satisfied in game-play. Everybody can find, say, the aesthetic value in the struggle to win, even if not everybody can win.

But, one might protest, this argument depends on a fairly primitive view of achievements that: they must be comparative. Perhaps, one might say, achievements should be fleshed out in terms of some non-comparative standard of perfection. Russell himself points to Robert Simon's discussion of mutualism, where we engage in competitive sports as part of a mutual support for our collective attempt to become more excellent: more skilled, more virtuous, more capable.

First, let me point out a subtle blurriness here. If the purpose of playing a game is to develop one's excellences, and the development of excellences is independent of winning (or success on some other standard provided by the game), then that counts as striving play. Recall: achievement play, in my account, is play where the value is bound up with winning, and striving play, in my account, is play where the value is bound up in the process of attempting to win. Crucially, the striving/achievement distinction is separate from the intrinsic/extrinsic value distinction (32-33). Striving play could be intrinsically valuable (we just love struggling in and of itself) or extrinsically valuable (struggling helps us become healthier, fitter, or develops our skills). We can tell that it's striving play, even if it's extrinsic, precisely because we can get what we want without winning. It doesn't matter if you finished last; running a marathon can still make you healthier, develop your grit, or what have you. The kind of play that Simon describes here is a kind of extrinsic striving play. And as I said in the book: when we play to develop each others' excellences in a non-opaque and unaltered way, that looked like coaching: we took as our direct and psychologically dominant goal the development of the others' excellence (176-7). But in many cases, the mutual development of excellence is a self-effacing goal. The best way to help develop another's excellence is to forget about that for a while, and just try to win. Thus: striving play is sometimes the best route to mutual excellence-development.

But I think there's something more I want to say here. Aesthetic and other forms of experiential play still have an advantage over excellence-centered play, even if that excellence-centered play occurs in a striving motivational structure. This is because, insofar as success in the quest for excellence is measured against some standard of perfection, not everybody will be able to achieve it. Standards of perfection don't adjust down. But aesthetic play can be adjusted to fit our various abilities. One of the most important aesthetic experiences of practical activity, for me, is that of the harmony of capacity - the harmony of finding my total abilities well-matched to the problem at hand (108-12). I'm a rock climber, but I have never been able to advance beyond a middling level; I apparently simply don't have a certain athletic explosiveness. My degree of 
achievement in rock climbing is capped. But my interest in elegant movement, in finding harmony between my abilities and the world, can be met simply by finding the kind of rock climbing problem that fits my abilities. I can find the harmony of capacity in climbing near the peak of my capacity, conquering climbs that are, by the standards of world-class athletes, barely warm-ups.

Interestingly, I should note that several philosophers of sport, in responding to this book, have been puzzled at this very feature of my account of the aesthetics of games: that novices and the lower skilled can derive as much aesthetic value from a game as the highly skilled. This puzzles them because, it seems to them, the most beautiful play should arise from the most skilled players. 6 But this gap is, in my mind, a feature and not a bug. The novice player may not look beautiful to spectators, but they can be having a beautiful experience of developing a new skill. The point of games is that, unlike the world, we can scale the challenges and obstacles to fit our abilities and interests. The world may be a grinding place which fits our capacities poorly, but we can find or engineer a game to fit nicely.

This capacity to pick the game that fits is, I think, something that many of my commentators have sometimes overlooked. For example, Patridge worries that the transparent game-player will be able to adjust themselves mid-play to suit the social purpose. The opaquely layered striving player, she implies, has no such capacity. As I've already argued, I do think the opaquely layered player has a certain degree of mid-game adaptability. But there's more to say. The opaquely layered player has another important tool: game choice. If our skills don't match well in this game, we can try and find a different game where they do. Patridge takes it as morally better that the transparent game-player with social ends will constantly monitor the other players and adjust their play-style to match. But one of the fascinating things for games, for me, is that, when the stars do align, they let us offload some of that moral maneuvering. When we have found a game we all enjoy, and players with the appropriate match of skill and spirit, we can get a moment of moral reprieve. For a little while, we don't have to worry much about how other people are doing and we can just play hard. Of course, as Morgan puts it, the stars do have to align. Such alignments are certainly not guaranteed. But if we value such moments of moral reprieve, we can put in the effort to align the stars. If the stars align only when we have people with similar motivational sets and skill levels, we can put efforts into finding those matches - by setting up environments like tournaments or conventions, which draw people with the right motivational sets. And such alignment can be made more approachable through structural features. Some game designs are more accommodating to people of different skill levels, or different skill types. And, as Jose Zagal and I argued elsewhere (2016), much of this alignment can be built into the social and technological environment. In online play, for example, systems that accurately rate

6 Russell expresses such puzzlement in his accompanying comments. In another set of comments, Tom Hurka has expressed a similar worry (Hurka, 2021). I have replied to Hurka at length elsewhere (Nguyen, 2021). 
players' skill levels, and then pair off players for matches with approximately similar skill levels, will do a lot of work towards that alignment.

Finally, Michael Ridge wants to suggest that my account of the value of striving play comes up short, because it leaves out a key concept: playfulness. In the book, I claim that games can help expand our autonomy. It does so when we lightly adopt the agential modes offered in games and shift easily between them. In that case, we are using games to explore different agential states and value perspectives. But other times, games can work to undermine our autonomy, by encouraging us to become stuck in a particular agential mode. What accounts for the fact that games can sometimes be autonomyexpanding, and other times autonomy-undermining? Ridge says that my account is insufficient, and suggests that the missing link is the attitude of playfulness.

According to Ridge, we have adopted the playful stance when we are poised for playful activity. We are engaged in playful activity "just in case the agent is engaged in unscripted activity for the fun of it". Ridge takes fun to be a cluster of affective, motivational, and perceptual states, including a sense of safety, a lack of anxiety, an enjoyment in incongruity, amusement, and more. What unites these various states is that "fun functions to motivate experimental behavior in safe environments". This is why, says Ridge, playfulness broadens our perspective. Playfulness is an essentially open-minded state. When we are playful, we are willing to consider options outside the norm, which helps to encourage the practice of reflection. When we engage with games playfully, we have a broader perspective. And the sense of safety involved in playfulness helps us to step back and be more reflective.7

My own account is far more minimal. In my discussion, we are not required to play games with an attitude of playfulness, in Ridge's sense, to get the autonomy-expanding effect. I tried to show how the bare structure of striving play does a significant amount of the work, without the need for any particular further attitude (216-22). Achievement play tends to encourage being stuck in one motivational frame because achievement play is precisely the mode where the in-game attempt to win connects with one's enduring

7 A further note on Ridge's discussion of how playfulness aids in aesthetic experience. There is a basic tension, in my account, between the demand that aesthetic attention be disinterested, and the essentially interested nature of gameplay. My solution to this tension is to say that we experience game-play from the stance of being absorbed in play while we're fighting to win -- as interested. But we can also take another stance - we can view our experience of absorption from a perspective outside of that instrumental absorption. For example, we can think retrospectively about our experience of practically struggling to win, and take on a disinterested attitude towards our entire experience. Ridge worries that my solution can't be right, because it involves the use of memory - that memory presents things as less vivacious and vibrant, and so can't be part of full aesthetic appreciation. He offers the attitude of playfulness as an alternate way to resolve the tension. Here, I part ways from Ridge, because I don't think there is a real tension between the use of memory and aesthetic appreciation. This is because I think that any temporally extended artwork requires the pervasive use of memory to aesthetically appreciate. Perhaps certain visual artwork can be apprehended immediately, in a moment (though I actually doubt this). But a novel, a symphony, an album, a movie - only tiny pieces of these works are experienced immediately. The assessment of the work as a whole requires the use of memory to assemble the parts, and admire the entire structure. More importantly, I think the use of retrospective memory is only one solution to dissolving the tension. Another is simply split-consciousness layering, as I've described above - where one part of you is engaged in the puzzles, and another is, at the same time, aesthetically appreciating the experience of the first part. 
values. Striving play, on the other hand, by its very structure intrinsically encourages movement between motivational frames. In striving play, we care intensely about winning during the game, but then we set that interest aside. Outside of the game, we reflect on the value of the game-playing activity in the light of our larger purposes. That basic structure - the very fact that striving play involves switching between these two evaluative perspectives - encourages reflectiveness. Unlike Ridge, I do not think an attitude of playfulness is required, only an evaluative switching. Evaluative can be done from any number of different attitudes.

I prefer the more minimal account because I suspect that many different motivational attitudes can drive us into striving play - and then essential structure of striving play drives us towards reflection. The basic motivational structure of striving has two stages. First, we evaluate means in terms of their relationship to our phenomenologically fixed interest in winning. Then, we evaluate the value of our interest in winning in terms of the value of our engagement in the activity of struggling to win. This basic structure is, by itself, supposed to encourage some degree of reflectiveness. I might take up sports for the sake of my mental well-being and for my spiritual rejuvenation. I could try becoming absorbed in various different sports, and then step back and reflect on whether they are adding to, or subtracting from, my sense of mental well-being. This encourages both an exploration of different agencies, and a reflection on them, for reasons having little to do with playfulness, in Ridge's sense. Importantly, striving play is a motivational structure based on the place where the value adheres, and not the type of value. You are engaged in striving play when you take on an interest in winning for some value to be found in the process. What that value is, is wide open. It is very important, in my account, that there is both intrinsically valued striving play (like the pure glory of the struggle itself) and extrinsically valued striving play (like the fitness and mental health benefits of the struggle). On my view - but not on Ridge's - engaging in extrinsically valued striving play for a purpose like mental health, rather than in a spirit of fun, still encourages that moment of stepping back from a well-defined activity, and reflecting on its value from a broader perspective.

So, in my account, the reflective advantage of striving play is supposed to apply to all striving play, performed for any reason. Some reasons are even more reflectivityencouraging than others, for reasons I describe in the book. I suggest that aesthetic striving play might be extra-conducive to reflection because of its sensitive, non-rulebound nature (219-22). I am happy to think that a playful attitude is another reflection booster. But my core point is that we don't need those extra attitudes. Notice that, on Ridge's view, non-playful, aesthetically oriented striving play would fail to have any autonomy-enhancing effect.

And think about all the reasons we might have for playing a game besides the fun of it, and all the attitudes under which we might strive, besides playfulness. We can play games for social connection, for brain training, because we are doing our job as a games 
reviewer, because they have been assigned in a class, or because we think they have some artistic merit and meaning. I take these motivations to be skew to fun. But if any of these motivations drive us into striving play, we will still turn that motivational corner. I need not play for fun in order to explore different agential modes, nor to practice moving between agential modes, nor to be driven to be reflective about the value of absorption in an agential mode. It almost seems like any value will do, so long as that value is embedded within that angled motivational structure of striving play.

In the end of the book, I do talk about cultivating a certain kind of playfulness something very much in line with Lugones' sense, in which playfulness involves holding a normative landscape with a certain lightness. (Note that this sense of playfulness is far more minimal than Ridge's.) But for Ridge, playfulness is a pre-requisite for the autonomyenhancing effect of games, where for me, playfulness is a possible consequence. In my account, playing games the right way has the potential to cultivate playfulness, even in those who lack it.

Similarly, it's not clear that unscripted action is required to encourage the exploration of different agencies, nor for the reflectiveness on the value of absorption in those agencies. First, if your notion of unscripted action involves having significant choices, then some games involve little to no agency at all. Many pure gambling games involve the loss of agency, and offer the experience of being just along for the ride, at the whim of fate. Second, some gameplay can be highly scripted. Think of learning to play a solved game (like tic-tac-toe, or now, checkers) or learning the scripts behind chess openings. Each of these is an activity that encodes an agential mode - one of following a script, or choosing between a limited set of scripts. Engaging in scripted striving play encourages movement into, movement out of, and then reflection on the value of the mindset of following scripts. And that reflection may reveal the upsides and downsides of scripted action. (And it's not all downside. My spouse, Melissa Hughs, says that one of the reason she loves yoga is that it's a wholly directed activity where, for once in her life, she doesn't have to make any choices for a whole luscious hour. It is not unreasonable to love, say, the peace involved in repeating the same yoga video every morning. You might discover, as Samuel Scheffler (2012) puts it, that repeating a regular script creates a habit, a ritual, which makes you a home in time that you can return to.)

Why might you think that unscripted action, engaged in out of playfulness, would be required for reflection and open-mindedness, and to reap the benefits for striving play? For one thing, Ridge suggests the right spirit of openness depends on a feeling of safety. But, says Ridge, when one truly cares about winning and losing, the anxiety of the potential loss can undercut the feeling of safety, which can, in turn, undercut our openness to new ideas. But here, again, Ridge has ignored here the contribution of layered agency. Striving play involves a motivational transition, so value reflection can occur from a different agential perspective than the one so absorbed in a desire to win. It is the achievement player for whom anxiety about winning can pervade their entire life, 
since their interest in winning is part of their enduring goals. But for the striving player, anxiety about winning or losing is confined to the game, and to the temporary agential construct adopted during the game. From their larger perspective, the striving player has no anxiety about winning or losing, because the interest in winning is merely a temporary, disposable end. The striving player may feel unsafe and anxious during the game, but those tensions should disappear when they set aside the temporary goals of the game. Outside the game, they can reflect without anxiety.

In order for game-playing to help us explore agential perspectives, we need not be reflective during the game itself. In fact, what I suggested in the book is that game-playing, at its best, involves an alternation between an unquestioned, unreflective, all-in-for-thewin mental style, and a more reflective one. This lets us see the values and limitations of the unreflective state, and hopefully offers us practice with fluidly moving in and out of the unreflective, all-out state.

\section{The aesthetics of agency}

The final cluster of comments concerns the aesthetics of agency.

First, some quick clarifications: my claim - that games are the art of agency - is often misread as saying that games are art when they give the players significant agency, or that games are artistically better when they give the players more agency. That is not, in any way, my view. My view is that games are the artform where designers manipulate features of agency in order to provide some valuable experience. Reducing somebody's choice-space or even eliminating players' ability to choose or act at all, counts as a manipulation of the features of agency, and can provide a valuable experience of agencies. And we can admire the artistry of some choice-limitation, as when I admire the extraordinary emergent strategic complexity arises from the tiny menu of options available to the player in limit hold 'em poker. Some games are great because of the spareness and the limitedness of the agency of offer. They are something like agency haiku.

Second, my claim that games are the art of agency is sometimes misread as saying that the aesthetic experience of games is, or should be, an aesthetic experience of agency - that a player's aesthetic focus should always be on the features of agency that have been manipulated by the game designer. This is in the background of Daniel Vella's criticisms of my account. Games, says Vella, give us experiences, not just of agency, but of all aspects of being - including boredom, inability, and claustrophobia. In fact, I agree entirely with Vella's description of the rich aesthetic experiences of being available in games. But I do not think his view is in tension with my own. My claim is that agency is the artistic medium in games, and not that the primary aesthetic experience in games is of agency. Game designers manipulates features of agency, as an artistic medium, to 
produce some effect. Sometimes, that effect involves directing attention towards the experience of agency. But other times, game designers manipulate agency to direct their players' attention somewhere else. Sometimes, game designers manipulate agency to focus players on their emerging social relationships, or an emerging invented narrative, or on a feeling of dread, horror and helplessness.8

An analogy: the medium of poetry is language. Some poetry draws attention to its use of its own use of the medium, drawing the reader's attention towards its skillful, potent, or dramatic use of language. But other times, the use of language recedes into the background, and the reader's attention is drawn to some non-linguistic subject matter, like the poet's sorrow, the look of fog rolling off water, or a memory of teenage awkwardness. Language is still the medium and the poet's use of it can still be extraordinarily skillful - but often the poet's use of the medium is self-effacing, sending the reader's attention somewhere else.

For example, one of my favorite haiku is this Issa poem, as translated into English by the poet Robert Hass:

Even with insects -

Some can sing,

Some can't!

Most readers, I suspect, will immediately be drawn to think about the truth of this observation. They may notice the poem's empathy with the natural world or feel a newfound sense of kinship with insects. These effects are accomplished through the careful use of language. It is very important that the poem starts with the phrase "Even with insects", which primes us to expect some surprising expansion into the insect world - in a way that is amusingly undercut by the simplicity and humility of the observation. (Compare the actual Issa/Hass poem with the following terrible variation: "Some insects can sing / some insects can't. / Hey, just like us!") Though a careful student of poetry might come to analyze how the poet's use of language creates that feeling of empathy and kinship, that's not where the poem directs our attention. My immediate experience of the poem is that it directs me to think about insects, humanity and our shared pathos, and not to its use of language.

8 Antonia Peacocke's discussion of the aesthetics of agency understands this point well, and runs with it to point out some of the phenomenology of aesthetic experiences supported by the use of the agential medium. I am entirely in agreement with the substance of her discussion. I would only like to point out that her discussion presents itself filling a gap in my account by introducing a discussion of the aesthetics of attention and affordances in games, where I take myself to have, at least briefly, already introduced those ideas in the book. For a discussion of the influence of the agential medium on attention, see my discussion of how our attention is directed by the goals of the game (118-9). For a discussion of the aesthetics of affordances, see my discussion of how affordances play into the medium of agency (1516). I should say that my brief discussion in the book of affordances doesn't represent their importance in games. I give them short shrift, mostly because they've been well-discussed in the extant literature on the aesthetics of games, where I think the manipulation of other features of agency — like our goals — has been relatively under discussed. 
A reasonable claim is that all poetry uses the medium of language. An implausible claim is that all poetry offers experiences solely of language. Similarly, my claim is only the (hopefully plausible) claim that agency is the medium of games. I am not making the (obviously false) claim that games offer experiences solely of agency.

Third, the claim that games are the art of agency is not supposed to preclude the possibility of sub-categories within the art of agency, or overlaps and hybrids with other mediums and other genres. Here I am thinking of Jon Pike's worries that my treatment of physical games and videogames under the same umbrella leaves out the particular relationship to embodiment in physical games. I am happy to allow that there is a profound difference between physical sports and boardgames, and between physical sports and videogames. But that doesn't mean that there isn't some useful higher-order category to study. What the history of aesthetics has shown is that we can get a huge amount out of thinking about particular genres - like thinking about the specifics of westerns, or film noir, or horror movies, or gothic novels, or mystery novels, or picaresques. But we can also learn something by thinking at very high levels of abstraction - about general features of the medium of film, or the medium of the novel, or narrative in general. And we can learn things about specific genres that cross-cut different media - like "detective stories", which occur in audio, film, television, and print - even if in each particular instantiation, an artwork's particularity is profoundly entangled with its particular medium.

But the proof is in the pudding. And hopefully the book itself is a demonstration of the insights might be gleaned from thinking of the art form of games at this highly general level. But let me offer a further anecdote in support of the relative usefulness of at least a little unified, generalized thinking about games.

I have spent this last summer learning to flyfish. At one point, in a fly shop, I overheard an argument between two flyfishers. Important background note: some flyfishers are purists, who will only fish with dry flies. This mean that all their fishing is done on the surface, with floating flies. The fish's strikes are wonderfully visible. But fish aren't always willing to eat surface flies, so other flyfishers will also use flies which sink below the surface, such as imitations of little nymphs. What I was overhearing was an argument between a dry fly purist - somebody who preferred to adhere to a dry-fly-only code in their own flyfishing - and a nympher - a flyfisher who was willing to use all methods, including subsurface nymphs.9 The nympher's argument was that the goal of flyfishing was to catch fish. Being willing to switch to nymphs was far more efficient, so the only rational way to flyfish was to use nymphs when appropriate. The dry fly purist's response was that simply that they just liked flyfishing more the dry fly way, even if they didn't catch more fish. They just liked the experience of casting dry flies more, and they liked the kind of breaks and pauses and harsh difficulties they experienced with dry fly purism.

9 Importantly, the purist here only held to the view that they preferred, for themselves, to fish only with dry flies. They did not hold the (far less defensible) position that everybody should only fish with dry flies. 
They liked the fact that when the dry flies weren't working well they had to hike further and search the water more carefully. And sometimes they caught no fish, but it just heightened the overall drama, no? The nympher couldn't see the point of that argument.

At this point, I couldn't help myself from intervening. (How could I possibly refrain?) I briefly explained Suits' view of games and suggested that the dry fly purist's devotion could be explained as an aesthetic preference for the kind of struggle which resulted from adhering to the further limitation of "dry flies only". The nympher, I said, was making the mistake of thinking that the goal of the game was the purpose. If, on the other hand, you thought that flyfishing was a (profoundly aesthetic) game, and the dry fly purist was making a choice about their preference in striving activities, then the dry fly purist wasn't being irrational at all. They were simply preferring a different game, with more restrictions.

I'm happy to say that the dry fly purist was utterly pleased by my analysis. (They even offered to take me fishing!) And the nympher could grudgingly accept the dry fly purist's choice as a rational one. And flyfishing is a sport, a deeply physical game whose pleasures and aesthetic joys arise from a profoundly embodied experience of motion, perception, rhythm, and delicacy. And two practitioners of the sport, at least, found an abstract analysis of the motivational structure of aesthetic striving games to have some explanatory utility, in thinking about their deeply physical game.

Let me now turn to two last criticisms. The first is J.S. Russell's, who worries that my account of striving play doesn't capture the full spectrum of gaming experiences, especially aesthetic experiences. I say that, in achievement play, you are trying to win for the value of winning, but in striving play, you are pursuing the win for the sake of the struggle. But, worries Russell, there are all sorts of other striving experiences that don't involve struggling. Consider the pleasures of playing a game that you are highly skilled at and succeeding easily. Consider, too, the value of carefully climbing a dangerous mountain under slow and careful control. These, says Russell, are not struggles, but they should be treated as part of striving. And, notes Russell, in many cases, struggles are not aesthetically pleasing; they are awkward and graceless. It is the easy wins that are often the most beautiful.

This, I think, is simply a misinterpretation of my claim. There are two possible senses of "struggle":

Bare struggle: a struggle is any attempt to overcome some obstacle

Difficult struggle: a struggle is an attempt to overcome some obstacle, in which the agent experiences some significant difficulty

In my description of striving play, I meant to use "struggle" in the bare sense and not the difficult sense. (Otherwise, it would be very hard to explain why I am so interested in 
the aesthetics of easy striving, which I describe in my discussion of easy harmonies (10910).) For some ears, my use may seem odd. I have learned that, at least for some readers, the colloquial use of "struggle" leans towards difficult struggles. I am happy to treat my use as a technical and stipulated, rather than natural. I had never intended the term "struggle" to necessarily indicate difficulty, awkwardness, or nearness to a capacity limit, but only to indicate the process of overcoming obstacles, rather than the end-point of having overcome them.

But here's a more important point to make here. There is also an aesthetics of difficult struggles. First, Russell and I do agree that there can be a comedic aesthetic to awkwardness. We differ, however, on is the importance of that category. Russell dismisses comic awkwardness as a minor and trivial part of game aesthetics, where I think these are significant. But more importantly: Russell thinks the awkwardness of being near our limit, in difficult struggles, will undercut their beauty, where I think that some forms of grace and beauty can be found in their most paradigmatic form in difficult struggles.

Here we need to sharply differentiate between the aesthetic experiences of spectators and the internal aesthetics of players. This is the key idea of my discussion of process aesthetics in Chapters 5 and 6 , and this exchange highlights why the discussion of process aesthetics is crucial to the book's big picture. The aesthetics of game spectatorship is a very different matter from the aesthetics of game playing. The aesthetics of game spectatorship is close kin to the aesthetics of theater, film, music, and traditional performance arts. The primary aesthetic qualities are in externally available aspects of the performance, which are then appreciated by a non-performing audience. In ballet, for example, what matters aesthetically is how the ballet looks to the spectator - not how it feels to the dancer.10 But in my account, in games, the primary aesthetic qualities occur in the player themselves. It is the player's experience of their own action, and not the appearance of their action to some external spectators, that is what really matters. And the player's inner aesthetic experience need not match their outer aesthetic display. To the external observer, a novice climber may look like graceless flailing. But the novice's hard-fought successes can feel glorious to the novice, themselves. The learning process can be full of epiphanic experiences of their own skill development, and of hardfought harmonies between their actions and the problem at hands. And their inner experience of their struggle is what matters in the process aesthetics of games.

On to the last piece of business. Chris Bartel offers a particularly biting set of criticisms of my discussion of aesthetics and artistic medium.11 In the book, I have mostly

10 I offer an extended discussion of this point in a new paper, "The Arts of Action", which extends and expands themes from the book's discussion of process aesthetics (Nguyen, 2020b).

11 Bartel quotes passages where I mention that I subscribe to a cluster theory of the arts, and reconstructs a version of an argument for games as an art, that uses a cluster theory of the arts. But in that passage, I am not trying to invoke a cluster theory of art as part of a formal argument that games are an art. I had tried to indicate that my mention of the cluster theory was only a passing comment, and not part of the formal argument of the book. In the end, I'm not too interested in plumping for any particular account of "art" or "aesthetic" and issuing a formal argument for games as fitting those accounts. Rather, I am interested in charting those ways in which games are similar to the traditional arts, 
waved my hands at the notion of "art" and refused to significantly argue for my claim that games are an art-form. Bartel is happy with this tactic; he and I are in agreement that the concept of "art" is less important than it's cracked up to be. But, he says, this causes me problems down the line, especially with my central claim that games work in the artistic medium of agency. But, says Bartel, artistic mediums only exist in art. It is precisely art where the medium matters so keenly and where we spend so much time thinking about the artist's use of the medium. The philosophy of art has been particularly interested in the medium-specificity of artistic content. That is: non-artistic content can be readily translated between different media. The same philosophical argument can be presented in text, speech, a YouTube video or a PowerPoint. The core content - the argument itself - translates between different media. But not so with art. An artwork's peculiar content is dependent on its precise use of a particular medium.

So here is how Bartel's criticism goes. According to Bartel, I don't have an good argument that games are art. But then I go on to talk about the artistic medium of agency, and the game-designer's use of that medium. But talking about the artistic medium only makes sense for things which we already know are art. So, since I've refused to establish that games are an art, then I have no grounds for talking about artistic mediums at all.

I actually want to flip this argument on its head and run it in exactly the opposite direction. Here's how that goes. Grant that art is distinctive in the fine use of artistic medium. It makes intuitive sense to talk about the game designer's use of the medium of agency. This gives us a reason to think that games are an art form. In short: if art is the only place where it makes sense to talk about mediums, then you shouldn't dismiss medium-talk about some candidate form by pointing out that we have yet to establish that the candidate under discussion is an art-form. Rather, the existence of sensible medium-talk about a form weighs in favor of its being an art.

And if you look at the rich history of game criticism and game appreciation, we find so much critical talk that is, in the light of my account, clearly concerns the medium of agency. "Oh, look how Miyamoto and his team set the exact friction in Super Mario Brothers. It helps create this incredible since of real physical movement when you're jumping".12 Or: "It's incredible how Knizia changed the entire feel of the game, by setting the victory condition in terms of how balanced your portfolio was, instead of the total value". Insofar as the appreciation of games involves appreciating how a game gets some desirable aesthetic effect through the manipulation of the agential medium - the goals, affordances, and practical environment of the in-game agent - then we have reason to think that that the appreciation of games is a kind of artistic appreciation.

\footnotetext{
and the ways in which they are different. I am fine, if you accept the substantive claims about the particularities here, if you decide that you would rather use some other terms than "art" or "aesthetic". But I waved in the direction of the cluster theory to explain why I use those terms - games are close enough to the traditional cluster for me. But those terms aren't the important thing; what I care about is getting right the specific qualities of our experience of games.

12 This is a paraphrase of a wonderful discussion by video game critic and designer Tim Rogers: http://www.actionbutton.net/?p=426
} 
The next question is, then: can games really be aesthetic? I have offered some reasons to think that our experience of games meets some of the traditional criteria for aesthetic experiences (114-20). But Bartel raises a new worry, which arises from the distinctiveness of my account of games as process art. If I had claimed that the aesthetic qualities occurred in the game itself, Bartel would have no quarrel. It would be unproblematic, for Bartel, if I had merely claimed that the aesthetic qualities lay in static and enduring qualities in the game design - like its rules, affordances, or graphics. But I say something entirely different. According to me, in games, the primary aesthetic effect emerges in the player themselves. It is the player who is graceful, brilliant, elegant, or comically inept. But, says, Bartel, the important thing is that, in the tradition of aesthetic philosophy, aesthetic properties adhere to objects. An aesthetic property isn't just in our experience of a thing, but it is in the thing itself. Aesthetic judgments aren't just reports of my inner experience. If I properly judge that a work is tragic, it isn't just my reporting that I feel a bit sad; it's me claiming that the novel itself is tragic.

Why might this be important, and what does it say about the status of games? I think there are two different aspects to why we might care about the objectembeddedness of aesthetic properties. First, we might care about the fact that there the aesthetic properties are perfectly public and sharable. In that aspect, I simply think games are different. Even when we play the same game, each of our distinct playings varies widely. If the quality of our particular playing performance is the real bearer of aesthetic properties, then you and I will never quite come into contact with the same aesthetic properties. I think this is simply a feature of games (and other process aesthetic practices), and an important difference to note.

But this concerns, not the actual separation of aesthetic experience and aesthetic object, but rather the publicity and sharability of the aesthetic object. As I read the history, however, the key feature in aesthetic properties is not sharability, but that that they occur in objects and so are independent of our experience of objects.13 That is, there is the painting, and then there is our experience of the painting. The beauty is in the painting, and not merely in our experience of it. To put it crudely, aesthetic qualities are not just in our subjective responses. It is this experience-independence that I think Bartel is most concerned with. However, this criterion of the aesthetic presents no problem for my account of game aesthetics. In my account or games as a process art, the aesthetic properties occur in my performance in playing a game, which different from my experience of my performance. In other words, if I climb a bouldering problem elegantly, that elegance is in my movement, in my solution - in my process of figuring things out. And that is very different from my experience of my movement, my experience of my solution, and my appreciative experience of how it felt to figure things out. Another way to put it: if I can admire the beauty in an extraordinarily elegant bit of climbing from Ashima Shiraishi - in the elegance of her movements and the quality of her solution - why

13 I offer a further discussion of the sharability standard and the object-embedded standard in Nguyen (2020b). 
can't she appreciate that beauty too? The only difference is that I think Shiraishi has a richer access to the elegant qualities of her movement than I do. But her experience of her movement is different from the movement itself. The beauty lies in the player's performance, and their performance is distinct from their experience of their performance.

I have one last argument on this point. I actually suspect this will be unsatisfying to most people, but it is what actually convinces me. Aesthetic properties like beauty, elegance, comedy, and drama - these are, according to the philosophical literature, accessible primarily through direct experience. I may never be able to define "elegance", or give you the principles by which something is elegant, but I recognize it when I see it. And I think, for many of us, the language that comes naturally to hand, when trying to describe our experience of our own actions and decisions in a game, is recognizably aesthetic language. My movement was beautiful, my climb was thrilling, my solution came from an incredibly elegant moment of my own mind. And I think, in many ways, there is no greater authority on what is aesthetic than the aesthetic perceiver themselves. If you find beauty in a sunset, and in Li Po's poetry, and also in your split-second decisions during a hard game of DoTA 2, then I, for one, am not going to argue with you.

\section{Bibliography}

Bratman, Michael. 1999. Intention, Plans, and Practical Reason. Cambridge, MA: The MIT Press.

Hurka, Thomas. 2021. "How Much Are Games Like Art?" Analysis. https://doi.org/10.1093/analys/anab017

Lugones, María. 1987. "Playfulness, 'World'-Travelling, and Loving Perception". Hypatia 2 (2): 3-19.

Nguyen, C. Thi. 2020a. Games: Agency as Art. Oxford: Oxford University Press.

Nguyen, C. Thi. 2020b. "The Arts of Action." Philosopher's Imprint 20 (14): 1-27.

Nguyen, C. Thi. 2021. "Defending Games: Reply to Hurka, Kukla, and Noë". Analysis. https://doi.org/10.1093/analys/anab047

Nguyen, C. Thi and Jose Zagal. 2016. "Good Violence, Bad Violence: The Ethics of Competition in Multiplayer Games." DiGRA/FDG '16 - Proceedings of the First International Joint Conference of DiGRA and FDG. http://www.digra.org/wpcontent/uploads/digital-library/paper 39.pdf

Scheffler, Samuel. 2021. "The Normativity of Tradition". In Equality and Tradition: Questions of Value in Moral and Political Theory. Oxford: Oxford University Press. 
